\title{
Reference values and correlations for multiple physical performance measures: a prospective cross-sectional study among independently mobile older men in Japan
}

\section{Yoshinori Ishii ( $\nabla$ ishii@sakitama.or.jp ) \\ ishii Orthopaedic \& Rehabilitation Clinic \\ Hideo Noguchi \\ Ishii Orthopaedic \& Rehabilitation Clinic \\ Junko Sato \\ Ishii Orthopaedic \& Rehabilitation Clinic \\ Hana Ishii}

Kanazawa Medical University, School of Plastic Surgery

Ryo Ishii

Sado General Hospital

\section{Seiya Kobayashi}

Ishii Orthopaedic \& Rehabilitation Clinic

\section{Takashi Iwamura}

Ishii Orthopaedic \& Rehabilitation Clinic

\section{Shin-ichi Toyabe}

Niigata University Graduate School of Medical and Dental Sciences

\section{Research article}

Keywords: Aging, bone quality, cognitive function, healthy life span, Japan, muscle strength, older adult, physical performance, reference value, walking ability

Posted Date: November 8th, 2019

DOl: https://doi.org/10.21203/rs.2.17063/v1

License: (c) (1) This work is licensed under a Creative Commons Attribution 4.0 International License. Read Full License 


\section{Abstract}

Background: As the most rapidly aging country in the world, Japan is one of few countries with a male life expectancy over 80 years. The gap between the healthy life span and the total life expectancy is large, highlighting the importance of maintaining physical performance (e.g., walking speed, grip strength, and standing balance) in later life, which is also negatively associated with mortality among older adults. The present study aimed to establish reference values for multiple physical performance measures among high-functioning oldest-old Japanese men and to investigate the correlations among these measurements to understand how these variables are related in a more detailed and objective way, compared with previous studies.

Methods: This single-center prospective cross-sectional study was conducted from September 2018 to August 2019 with 120 male orthopedic patients in Japan who were able to walk independently. Seven measures of physical performance were assessed: handgrip strength, quadriceps strength, static balance ability (one-legged stance), dynamic balance ability (Functional Reach Test; FRT), walking ability (5-m walking time test), combined movement ability (Timed Up \& Go test), and bone quality. Cognitive function was also measured (Mini-Mental State Examination; MMSE). Spearman's rank correlation was used to evaluate correlations between the variables.

Results: Specific reference values are reported for each physical performance measurement explored in this study. Only six participants were classified as cognitively impaired, and 16 had mild cognitive impairment. There were significant correlations of varying levels among all of the measures of physical performance. Age was significantly correlated with all performance measures except FRT, and there was no correlation between age and MMSE. MMSE was weakly correlated with FRT and unrelated to the other performance measures.

Conclusions: The reference ranges from this study among a high-functioning cohort of men aged 80 years or older can be used by older men who have not yet reached this age and their health care providers as physical performance targets to facilitate the maintenance of independent mobility in later life. There is an urgent need for the establishment of exercise interventions that will allow these men to achieve the desired targets, potentially lengthening their healthy life span.

\section{Background}

According to the Ministry of Health, Labour and Welfare of Japan, the average life expectancy in Japan in 2018 was 81.25 years for men and 87.32 years for women, both of which were record highs [1]. The healthy life span (period during which there is no restriction on daily life caused by mobility limitations) was 72.14 years for men and 74.79 years for women as of 2016 [1]. This shows that there is a large gap between the life expectancy and the healthy life span. Several previous studies have reported a link between poor physical performance and mortality. Nofuji et al. [2] found that lower scores on several measures of physical performance, such as walking speed, grip strength, and standing balance, were 
associated with mortality in a general population of older adults in Japan. In a population-based cohort study in England, Nüesch et al. [3] reported a positive association between the severity of walking disability and the risk of death among patients with knee or hip osteoarthritis. In another cohort study, Imura et al. [4] reported that postoperative ambulatory level at hospital discharge reliably predicted survival rate among older adults after hip fracture. Considering these previous findings, improving physical performance among older adults may lead to reduced mortality, both individually and at the population level in aging countries such as Japan. Therefore, reference values for measures of physical performance among older adults who are able to walk independently should be clarified for use as target values, both to shorten the gap between the life expectancy and the healthy life span and to extend life expectancy.

However, only a few reports $[5,6]$ have conducted simultaneous evaluations of multiple physical performance measures and tested the correlations among these measures. Previous studies have included one or two physical performance measures and have usually been conducted among community-dwelling older adult men and women of various ages (i.e., in their 60 s, 70 s, or 80 s and older) and with different levels of walking ability [7-15]. Most of these studies have evaluated the correlations between cognitive function $[5,7,8,11,12,13]$ or age $[6,9,10,11]$ and measures of physical performance; few studies [16] have reported the correlations among performance measures for older adults. Measuring multiple types of physical performance simultaneously and examining the correlations among these measures will allow us to evaluate the characteristics of these measures and the links among them in a more detailed and objective way, compared with existing work.

According to recent reports, older age is related to higher mortality rates after total knee arthroplasty (TKA) among patients aged over 75 years [17] and after hip fracture surgery, with mortality rates for these patients observed to be over two times higher among men aged over 80 years than among men aged 6569 years [18]. Notably, there are only 11 countries with a male life expectancy above 80 years, although this is more common for female life expectancy [1]. In terms of gender differences in physical performance, a few previous studies have reported different patterns of age-related decline for men and women $[6,9,11]$. In addition, functional recovery after traumatic hip fracture has been found to be better among men than among women [19]. Conversely, the mortality rate after hip fracture was found to be higher for men than for women $[18,20]$. Considering these previous findings, assessing physical performance among men aged 80 years or older who are able to walk independently might facilitate improvements in men's life span.

Therefore, this study aimed 1) to establish reference values for multiple physical performance measures that have been reported to be related to the level of ambulation and the risk of falling among men aged 80 years or older who are able to walk independently and 2) to assess the correlations among seven measures of physical performance, as well as these measures' correlations with age and cognitive function, which have been examined in previous studies [5-13]. In terms of the clinical significance of this work, older adults aged under 80 years may be able to use the reference values established in this study as targets, facilitating the maintenance of independent walking as they age. For this reason, and in 
contrast to previous studies of community-dwelling older adults, the main objective of the present study was to define reference ranges for physical performance variables among a high-functioning cohort.

\section{Methods}

This prospective cross-sectional study was conducted from September 2018 to August 2019. The study included data on 120 Japanese male patients who visited an orthopedic specialty outpatient clinic (median age: 84 years, $25^{\text {th }}$ percentile: 81 years, $75^{\text {th }}$ percentile: 86 years, range: $80-94$ years). These patients received diagnoses of degenerative joint and/or cartilage disease. More specifically, the diagnosis was spine-related for 58 patients, upper limb-related for 14 patients, lower limb-related for 43 patients, and related to other areas for 5 patients. To be included in the study, patients had to be men aged 80 years or older who were able to walk independently without support from others, with or without a T-cane. The exclusion criteria were as follows: 1) neurological findings, such as motor paralysis; 2) cognitive or mental dysfunction requiring medication; or 3) skeletal dysfunction that had a negative impact on walking. This study was conducted based on the guidelines laid down in the Helsinki Declaration and the ethical guidelines of our institution. The Research Board of Healthcare Corporation Ashinokai, Gyoda, Saitama, Japan, approved the study (ID number: 2018-5). All patients provided written informed consent for participation. The patients' background characteristics are shown in Table 1.

\section{Physical performance measures}

\section{Muscle strength}

Upper extremity: handgrip strength. Handgrip strength (GS) was measured in the dominant hand using a digital dynamometer (T. K. K.5401 GRIP D, Takei Scientific Instruments Co., Ltd., Niigata, Japan) (minimum measurement unit: $0.1 \mathrm{kgf}$, accuracy: $\pm 2.0 \mathrm{kgf}$ ). Participants were instructed to inhale deeply and fully exhale while squeezing the dynamometer with as much force as possible with their dominant hand. The best result of two trials was used for the analysis. This value was divided by body weight (BW) in $\mathrm{kg}$ to eliminate the effect of physique. This ratio of muscle strength (MS) to BW (MS/BW ratio; $\mathrm{kgf} / \mathrm{kg}$ ) was also used in the analysis.

Lower extremity: quadriceps strength. To assess quadriceps strength (QS), isometric knee extension MS in $\mathrm{N}$ was measured with the knee in approximately $20^{\circ}$ of flexion using a Locomo Scan dynamometer (Alcare Corp., Tokyo, Japan), following a standard protocol [10]. Two measurements of QS were taken, and the highest value on each side was used in the analysis. These values were divided by BW to eliminate the effect of physique. The resulting ratio of MS to BW (MS/BW ratio; N/kg) was used in the analysis. For the assessment of the correlations among the performance measures, for each participant, the side with the highest MS/BW ratio was used.

\section{Body balance}


Static balance ability with eyes open. One-legged stance (OLS) with eyes open was assessed using the participant's preferred leg. Participants were asked to place their hands on their waists while staring at a mark on the wall, raise one leg, and stand for as long as possible. They were timed until they lost their balance or reached the maximum time of 60 seconds. Participants performed two trials, and the longer time (to the nearest 0.1 second) was used in the analysis.

Dynamic balance ability. The Functional Reach Test (FRT) was used to measure dynamic balance ability. Each participant was instructed to lift his preferred upper limb to a horizontal position and to reach as far as possible without taking a step or touching the wall. During this assessment, the participants' elbows were extended, their forearms were in neutral pronation/supination, and their hands were open with fingers extended. The initial and maximal reach were measured in $\mathrm{cm}$ from the end of the third finger using a meter stick. The measure was performed twice, and the longer of the two reaches was used in the study.

\section{Movement ability}

Walking ability. The 5-m walking time test was used to assess walking ability. This test was conducted as an in-room test consisting of an 11-m linear walking exercise comprising an initial 3-m acceleration zone, a central 5-m timed zone, and a final 3-m deceleration zone. The 5-m walking time started when the patient's lower limb crossed the starting line between the acceleration zone and the 5-m timed zone and ended when the patient crossed the end line between the 5-m timed zone and the deceleration zone. The measures taken were the time it took the patient to walk the entire 5-m zone at normal and maximum speed (walking time in seconds), as well as the distance $(5 \mathrm{~m}$ ) divided by the walking time (walking speed; $\mathrm{m}$ /second). In this study, both normal walking speed (5-m NWS) and maximum walking speed (5$\mathrm{m}$ MWS) were used in the evaluation of correlations among the performance measures.

Combined movement ability. The Timed Up \& Go (TUG) test was used to measure combined movement ability. For this test, participants were instructed to stand from a seated position in a chair, walk $3 \mathrm{~m}$, turn, walk back, and sit down in the chair again as quickly as they could. The measure taken from the TUG test was the total time in seconds it took for a participant to complete the test and return to the starting seated position.

\section{Bone quality}

Bone quality was assessed by bilateral calcaneus broadband ultrasound attenuation (BUA; $\mathrm{dB} / \mathrm{MHz})$ using an AOS-100SA (Hitachi-Aloka Medical, Ltd, Tokyo, Japan). All measurements were taken with the same device. Two transducers (receiving and emitting) faced with rubber coupling pads were placed in direct contact on either side of the patient's heel. Ultrasound gel was applied to the coupling pads to ensure good contact. Standard protocols for this instrument were used to obtain the BUA, including calibration and positioning of the participants. For each participant, the higher value of the two sides was used in the study. The intraclass correlation coefficient for calcaneus BUA, calculated to assess testretest reliability, was 0.988 (95\% confidence interval: 0.955-0.997). 
We evaluated the patients' level of cognitive functioning quantitatively using the Mini-Mental State Examination (MMSE). This widely used screening test for mental status is designed to evaluate orientation, registration, recall, and attention in older adults. Scores range from 0 to 30 points. The patients were classified as cognitively impaired ( $<20$ points) or not cognitively impaired ( $\geq 20$ points) [21]. In addition, mild cognitive impairment ( $\mathrm{MCl})$ was defined as scoring 23 points or less [22].

\section{Statistical analysis}

Data for certain variables did not pass the Kolmogorov-Smirnov or Shapiro-Wilk test of normality. We used the Wilcoxon signed-rank test to determine whether there was a left-right difference in continuous variables. We used Spearman's rank correlation test to evaluate correlations between variables. The strength of the correlation of the rank coefficients was defined as strong $(0.70-1.0)$, moderate $(0.40-$ $0.69)$, or weak $(0.20-0.39)$ [23]. In all tests, a $p$ value $<0.05$ was considered statistically significant. All statistical analyses were performed with IBM SPSS Statistics, Version 23 (IBM Japan, Tokyo, Japan). Values are expressed as medians (25th, 75th percentile; range).

\section{Results}

The median MMSE score was $26(25,29 ; 16-30)$ (Table 1$)$. Six participants were regarded as cognitively impaired (scoring $<20$ points), and 16 participants had $\mathrm{MCl}$.

With regard to MS, we explored both absolute values and weight-adjusted ratios. The median GS was 29 $\mathrm{kgf}(25,33 ; 12-45)$, and the median MS/BW ratio was $0.49 \mathrm{kgf} / \mathrm{kg}(0.40,0.55 ; 0.24-0.69)$ (Table 1$)$. For the right side, the median QS was $302 \mathrm{~N}(229,359 ; 82-679)$, and the median MS/BW ratio was $4.9 \mathrm{~N} / \mathrm{kg}$ $(3.8,6.1 ; 1.4-10.9)$; for the left side, these measures were $312 \mathrm{~N}(241,382 ; 91-669)$ and $5.3 \mathrm{~N} / \mathrm{kg}(3.9$, $6.4 ; 1.5-0.1)$, respectively. There was no statistically significant difference between sides $(p>0.05)$ (Table 1).

The median static balance ability, as assessed through OLS with eyes open, was 14 seconds $(5,39 ; 1-$ 60). Dynamic balance ability, measured using the FRT, had a median value of $27 \mathrm{~cm}(21,31 ; 5-48)$ (Table 1).

Five-meter normal and maximum walking ability were measured in relation to both time and speed. The median 5 -m NWS was $1.2 \mathrm{~m} / \mathrm{second}(0.9,1.3 ; 0.3-1.9)$, and the test took 4 seconds $(4,6 ; 3-19)$. The median 5 -m MWS was $1.5 \mathrm{~m} /$ second $(1.2,1.8 ; 0.3-2.8)$, and the test took 3 seconds $(3,4 ; 2-6)$. The median TUG test score, which was included to assess combined movement ability, was 8 seconds $(7,10$; 4-23) (Table 1).

The median BUA was $57 \mathrm{~dB} / \mathrm{MHz}(47,64 ; 23-109)$ in the right calcaneus and $56 \mathrm{~dB} / \mathrm{MHz}(44,65 ; 16-94)$ in the left calcaneus, and there was no statistically significant difference between the two sides $(p>0.05)$ (Table 1). 
With regard to the associations among the measures of physical performance, there were significant correlations of varying levels for all the measures (Table 2). A strong positive correlation was observed between 5-m MWS and 5-m NWS ( $p<0.0001, r=0.847)$, and a strong negative correlation was observed between 5-m MWS and the TUG test $(p<0.0001, r=-0.776)$ (Table 2). Both measures of MS (GS and QS) showed weak positive correlations with static (OLS) and dynamic (FRT) body balance (GS adjusted for body weight vs. OLS: $p<0.0001, r=0.380$; GS adjusted for body weight vs. FRT: $p<0.0001, r=0.339$; QS adjusted for body weight vs. OLS: $p<0.012, r=0.229$; QS adjusted for body weight vs. FRT; $p<$ $0.0001, r=0.329$ ) (Table 2 ). The other physical performance measures were moderately correlated with each other (Table 2). TUG test score was negatively correlated with the other measures of performance; the other performance measures were positively correlated with each other (Table 2).

We observed positive or negative correlations between age and all performance measures except FRT, and there was no correlation between age and MMSE. The only correlation observed between MMSE and a performance measure was a weak correlation with FRT (Table 2).

The median MMSE score was $26(25,29 ; 16-30)$ (Table 1). Six participants were regarded as cognitively impaired (scoring $<20$ points), and 16 participants had $\mathrm{MCl}$.

With regard to MS, we explored both absolute values and weight-adjusted ratios. The median GS was 29 $\mathrm{kgf}(25,33 ; 12-45)$, and the median MS/BW ratio was $0.49 \mathrm{kgf} / \mathrm{kg}(0.40,0.55 ; 0.24-0.69)$ (Table 1$)$. For the right side, the median QS was $302 \mathrm{~N}(229,359 ; 82-679)$, and the median MS/BW ratio was $4.9 \mathrm{~N} / \mathrm{kg}$ $(3.8,6.1 ; 1.4-10.9)$; for the left side, these measures were $312 \mathrm{~N}(241,382 ; 91-669)$ and $5.3 \mathrm{~N} / \mathrm{kg}(3.9$, $6.4 ; 1.5-0.1)$, respectively. There was no statistically significant difference between sides $(p>0.05)$ (Table 1).

The median static balance ability, as assessed through OLS with eyes open, was 14 seconds $(5,39 ; 1-$ $60)$. Dynamic balance ability, measured using the FRT, had a median value of $27 \mathrm{~cm}(21,31 ; 5-48)$ (Table $1)$.

Five-meter normal and maximum walking ability were measured in relation to both time and speed. The median 5 -m NWS was $1.2 \mathrm{~m} /$ second $(0.9,1.3 ; 0.3-1.9)$, and the test took 4 seconds $(4,6 ; 3-19)$. The median 5-m MWS was $1.5 \mathrm{~m} / \mathrm{second}(1.2,1.8 ; 0.3-2.8)$, and the test took 3 seconds $(3,4 ; 2-6)$. The median TUG test score, which was included to assess combined movement ability, was 8 seconds $(7,10$; 4-23) (Table 1).

The median BUA was $57 \mathrm{~dB} / \mathrm{MHz}(47,64 ; 23-109)$ in the right calcaneus and $56 \mathrm{~dB} / \mathrm{MHz}(44,65 ; 16-94)$ in the left calcaneus, and there was no statistically significant difference between the two sides $(p>0.05)$ (Table 1).

With regard to the associations among the measures of physical performance, there were significant correlations of varying levels for all the measures (Table 2). A strong positive correlation was observed between 5-m MWS and 5-m NWS ( $p<0.0001, r=0.847)$, and a strong negative correlation was observed 
between 5-m MWS and the TUG test $(p<0.0001, r=-0.776)$ (Table 2). Both measures of MS (GS and QS) showed weak positive correlations with static (OLS) and dynamic (FRT) body balance (GS adjusted for body weight vs. OLS: $p<0.0001, r=0.380$; GS adjusted for body weight vs. FRT: $p<0.0001, r=0.339$; QS adjusted for body weight vs. OLS: $p<0.012, r=0.229$; QS adjusted for body weight vs. FRT; $p<$ $0.0001, r=0.329$ ) (Table 2 ). The other physical performance measures were moderately correlated with each other (Table 2). TUG test score was negatively correlated with the other measures of performance; the other performance measures were positively correlated with each other (Table 2).

We observed positive or negative correlations between age and all performance measures except FRT, and there was no correlation between age and MMSE. The only correlation observed between MMSE and a performance measure was a weak correlation with FRT (Table 2).

\section{Discussion}

The most important contribution of this study was the simultaneous evaluation of seven physical performance measures among Japanese men aged 80 years or older who were able to walk independently, revealing the reference values for these measures in this study population. Following previous studies, we also evaluated the correlations between cognitive function and physical performance $[5,7,8,11,12,13]$ and between age and physical performance $[6,9,10,11]$. To the best of our knowledge, the present study is the first to examine correlations among multiple physical performance measures, finding various levels of correlation. We found negative correlations between age and all performance measures except FRT, and age was not correlated with MMSE. MMSE was found to be weakly correlated with FRT and unrelated to the other six performance measures.

Cognitive status is considered a predictive factor for healthy life span among older adults-especially in terms of physical activity and walking level [24-26]. Matsueda et al. [25] reported a significant relationship between walking level and cognitive status evaluated by the MMSE among patients with hip fracture: Significant differences were observed in mean MMSE by walking status among people in their 80s (dependent walking status: 5.9 points, partially dependent walking status: 16.5 points, independent walking status: $23.7 ; p<0.0001)$. In the present study, all participants were able to walk independently, and 95\% (114/120) were categorized as not cognitively impaired using the MMSE. Taken together, our findings along with those from previous reports indicate that cognitive status can be viewed as an important predictor of maintaining physical activity levels among men aged 80 years or older. However, we did not find significant correlations between MMSE and most of the examined physical performance measurements. This is in contrast to the many studies that have reported significant correlations between cognitive function and aspects of physical performance, such as GS [12,13], QS [7], and walking speed $[5,27]$. These conflicting results may be explained by the fact that these previous studies included participants with various degrees of physical performance ability, whereas the present study evaluated only participants who were able to walk independently and therefore had a relatively high level of physical performance. Therefore, most participants in our study could be expected to also have relatively high MMSE scores because of their high level of physical performance. This focus on only participants 
with high levels of physical performance and cognitive ability may have made it difficult to detect a significant association between MMSE and the physical performance measurements.

With regard to MS, GS and QS have been reported to decline with aging $[6,9,11,28]$. Likewise, we found weak negative correlations between aging and both GS and QS. Seino et al. [6] reported the mean GS as $27.7 \pm 5.9$ and the mean weight-adjusted ratio as $0.49 \pm 0.11$ for those aged $80-84$ years; for those aged $\geq 85$ years, these researchers reported these values to be $23.2 \pm 5.3$ and $0.45 \pm 0.10$, respectively. In addition, a few previous studies $[28,29]$ have evaluated QS using the same arthrometer used in this study. Ishii et al. [29] previously reported that the median MS/BW ratio for QS was $3.3 \mathrm{kgf} / \mathrm{kg}$ for patients with posterior cruciate ligament-retaining TKA, $3.4 \mathrm{kgf} / \mathrm{kg}$ for patients with posterior cruciate ligamentsubstituting TKA (median age: 81 years), and $4.6 \mathrm{kgf} / \mathrm{kg}$ for controls (median age: 83 years). For Japanese men aged 80-89 years $(n=86)$, another study reported the median MS/BW ratio for QS to be $5.9 \mathrm{kgf} / \mathrm{kg}$ [28]. The values for both GS and QS found the present study were consistent with these previous findings. We believe that the index values of both GS and QS reported in the present study can be used as target values for adults aged under 80 years to maintain independent walking ability as they age.

One-leg balance is an important predictor of injurious falls in older persons [15], and a systematic review has demonstrated that it can also be a predictive tool for frailty among community-dwelling older adult populations [30]. For individuals aged $>75$ years, Seichi et al. [31] proposed a cutoff of 6 seconds for average OLS time to screen older adults for medical interventions or training programs. Seino et al. [6] reported mean OLS values of 26.0 seconds for those aged 80-84 years and 21.9 seconds for those aged 85 years or older. Harato et al. [32] reported 8.7 seconds as the mean OLS among patients graded as Kellgren-Lawrence grade IV [33] before TKA. Taking these previous results into account, the participants in the present study can be considered to have maintained relatively good one-leg balance and to be at low risk of falls.

The FRT is commonly used as an indicator of dynamic balance in older adults. Kamide et al. [34] concluded that older age and female sex were negatively associated with FRT value and that height and two-arm reach were positively associated with FRT value. In this study, because FRT was the only indicator of the seven performance measures that was not correlated with age, it was speculated that FRT was influenced more by other factors, such as physique, height, and two-arm reach, than by age. A recent meta-analysis of data from 20 published studies reported that the weighted mean (standard error) of FRT in these previous studies was $27.2(0.9) \mathrm{cm}$, which provides a reasonable standard for interpreting FRT performance among community-dwelling older adults [35]. Considering the findings of these previous studies, the median FRT value of $27 \mathrm{~cm}$ found in the present study can be considered to indicate that the study participants maintained satisfactory dynamic balance.

With regard to walking ability, previous studies $[5,6,9,27,36]$ have focused on walking speed. Recently, Grande et al. [37] summarized the existing evidence concerning the associations of slow gait speed with cognitive decline and dementia, drawing on 39 previous studies. Maximum gait speed may be the best 
walking-ability marker for cognition among older adults [27]. Seino et al. [6] reported mean values of 1.16 $\mathrm{m} /$ second for usual gait and $1.73 \mathrm{~m} /$ second for maximum gait among community-dwelling people in Japan aged 80-84 years; for those aged 85 years or older, these values were $1.11 \mathrm{~m} /$ second and 1.65 $\mathrm{m} /$ second, respectively. Amano et al. [38] identified sex, age, and Kellgren-Lawrence grade [33] as factors influencing walking ability such as 5-m walk test and TUG test outcomes. In the present study, both walking time and walking speed fell within the ranges presented in previous reports $[2,5,9,27,36,38]$. We believe that our results of relatively high values for MMSE and walking speed, even among men aged 80 years or older may support the previously reported positive correlations between cognitive level and physical performance [27,37].

The TUG test is a commonly used screening tool to assist clinicians in identifying patients at risk of falling. This test is recommended for the routine screening for risk of falls in the guidelines published by the American Geriatric Society and the British Geriatric Society [39]. However, a systematic review of diagnostic accuracy [40] suggests that a single assessment tool such as the TUG test should not be used to identify community-dwelling older adults at increased risk of falls. Whether there is a significant correlation between TUG test score and falling remains a controversial topic. However, the TUG test might be helpful in detecting cognitive impairment: The mean TUG test time has previously been reported as 13.2 seconds among people in their $80 \mathrm{~s}$ without $\mathrm{MCl}$ [8]. Taniguchi et al. [14] reported a mean TUG test score of $9.0 \pm 4.1$ seconds for community-dwelling older adults in their $80 \mathrm{~s}$. The average TUG test values found in the present study were higher than these previous findings. This discrepancy may be explained by the fact that the participants in the present study might be at low risk of falling because few participants with cognitive impairment were included.

BUA is recognized as a parameter associated not only with bone density but also with bone architecture and elasticity [41]. Ultrasonographic measurements of the os calcis have been shown to predict the risk of hip fracture among older women with equal accuracy to dual-energy x-ray absorptiometry of the hip [41]. Ishii et al. [42] reported a significant improvement in BUA 5 years after TKA surgery, concluding that this improvement might be explained by increased activity levels after surgery, which might increase mechanical loading of the calcaneus among older adult patients. Yanagimoto et al. [43] used BUA to demonstrate that the amount of walking was positively correlated with bone quality in older adults. In addition, Yung et al. [44] reported that weight-bearing exercises had positive effects on calcaneal bone properties, as assessed using quantitative ultrasound (including BUA). We believe that men aged 80 years or older who are able to walk independently may maintain good bone quality because of their activity levels.

The present study revealed significant correlations of varying levels among all measured performance variables. Because walking independently requires people to adjust their center of gravity while maintaining good body balance, all physical performance measures in this study should be effectively linked to accomplish this task. If even one of the seven types of physical performance was not wellfunctioning, we believe older adults might not accomplish independent walking. In fact, a previous report [13] found that even grip strength, which is often thought to be unrelated to walking ability, affects the 
level of activities of daily living for people aged 80 years or older. In terms of the different strengths of the correlations among the performance measurements, it seems reasonable that walking speed was strongly correlated with the other variables. It also seems reasonable that participants with a fast 5-m NWS would also have a fast 5-m MWS, leading to a positive correlation. Likewise, a fast 5-m MWS was linked to a short TUG time, resulting in a negative correlation between these measures. However, it is difficult to determine the reasons for the differences between weak and moderate correlations among performance measurements in the present study. These differences should be clarified using a larger number of cases in multicenter studies in the future.

This study had several limitations. First, the participants who took part in the study suffered from some skeletal dysfunction caused by degenerative joint and/or cartilage disease. However, because they did not need the support of others to walk, the impact of their disease status on their physical performance seemed to be negligible or small. Conducting our study in this clinical setting also meant that some equipment that is not usually available in research could be used to evaluate QS and bone quality. This allowed us to add two rarely explored physical performance measurements to this study. Second, the total number of participants was lower than the samples used in previous studies of community-dwelling older adults $[6,9,16]$. However, the number of participants who were men aged 80 or older was comparable to the samples of this population subgroup used to evaluate physical performance in previous studies, where the number participants in this age group has ranged from 9 to $68[8,14,28,30,31]$. Third, we did not consider other factors that may impact the physical performance measures, such as age, sex, and race. Finally this was a single-center study, which may limit the generalizability of the results. Verification of the validity of our results through research at multiple facilities is expected in the future.

Despite these limitations, this is a valuable report showing reference values for the highest number of physical performance measurements to date (previous work has examined a maximum of six physical performance measurements $[5,6])$, as well as the correlations among them, for 120 men aged 80 years or older who were able to walk independently in one of the most rapidly aging nations in the world. The reference ranges found in the present study can be used by older adults who have not yet reached the age of 80 years and their health care providers as target values to facilitate the maintenance of independent walking.

\section{Conclusions}

The present study evaluated physical performance measurements that are related to walking and falls among men aged 80 years or older who were able to walk independently. The study clarified the reference values of these variables, as well as the correlations among them, in this population. The reference values reported in this study can be used as target values for older adults aged under 80 years for maintaining physical performance as they age. Establishing an efficient exercise regimen to achieve these target values seems to be an urgent issue for older adults who fall short of the reference values for the examined indicators of physical performance.

Page $11 / 18$ 


\section{List Of Abbreviations}

TKA: total knee arthroplasty

MCl: mild cognitive impairment

MMSE: Mini-Mental State Examination

MS: muscle strength

BW: body weight

GS: handgrip strength

QS: quadriceps strength

OLS: one-legged stance

FRT: Functional Reach Test

WT: walking time

5-m NWS: 5-m normal walking speed

5-m MWS: 5-m maximum walking speed

TUG: Timed Up \& Go test

BUA: broadband ultrasound attenuation

\section{Declarations}

Ethics approval and consent to participate

All procedures in this study that involved human participants were performed in accordance with the ethical standards of the Research Board of Healthcare Corporation Ashinokai, Gyoda, Saitama, Japan, approved the study (ID number: 2018-5) and with the 1964 Helsinki declaration and its later amendments or comparable ethical standards. Written informed consent was obtained from all individual participants included in the study.

Consent for publication

Not applicable.

Availability of data and materials 
The datasets used and/or analyzed during the current study are available from the corresponding author on reasonable request.

\section{Competing interests}

The authors declare that they have no competing interests.

\section{Funding}

This work was partially supported by the Japanese Clinical Orthopedic Association under Grant 2019. The funder played no role in the design, methods, subject recruitment, data collection, analysis or preparation of the paper.

\section{Authors' contributions}

YI contributed to the study conception and design, drafted the article, and ensured the accuracy of the data and analysis. $\mathrm{HN}$ and JS contributed to the study conception and design and to the analysis and interpretation of the data. HI, RI, SK, and TI contributed to the data collection. ST provided statistical expertise and contributed to ensuring the accuracy of the data and analysis. All authors approved the final manuscript.

\section{Acknowledgments}

We would like to thank all the rehabilitation staff, all the interviewers, and most sincerely all the participating patients who visited our outpatient clinic. We also thank Jennifer Barrett, PhD, from Edanz Group (www.edanzediting.com/ac) for critically reviewing and editing a draft of this manuscript.

\section{Authors' information}

${ }^{1}$ Ishii Orthopaedic \& Rehabilitation Clinic, 1089 Shimo-Oshi, Gyoda, Saitama 361-0037, Japan

${ }^{2}$ Kanazawa Medical University, School of Plastic Surgery, 1-1 Daigaku Uchinada, Ishikawa 920-0253, Japan

${ }^{3}$ Sado General Hospital, 161 Chikusa Sado, Niigata 952-1209, Japan

${ }^{4}$ Niigata University Crisis Management Office, Niigata University Hospital, Niigata University Graduate School of Medical and Dental Sciences, 1 Asahimachi Dori Niigata, Niigata 951-8520, Japan

\section{References}

1. Ministry of Health, Labour and Welfare of Japan https://www.mhlw.go.jp/index.html

2. Nofuji Y, Shinkai S, Taniguchi Y, Amano H, Nishi M, Murayama H, et al. Associations of Walking Speed, Grip Strength, and Standing Balance With Total and Cause-Specific Mortality in a General 
Population of Japanese J Am Med Dir Assoc. 2016;17(2):184.e1-7.

3. Nüesch E, Dieppe P, Reichenbach S, Williams S, Iff S, Jüni P. All cause and disease specific mortality in patients with knee or hip osteoarthritis: population based cohort study. BMJ. 2011;342:d1165.

4. Imura K, Ishii Y, Yagisawa K, Matsueda M. Postoperative ambulatory level after hip fracture in the elderly predicts survival rate. Arch Orthop Trauma Surg. 2000;120(7-8):369-71.

5. Abe T, Soma Y, Kitano N, Jindo T, Sato A, Tsunoda K, et al. Change in hand dexterity and habitual gait speed reflects cognitive decline over time in healthy older adults: a longitudinal study. J Phys Ther Sci. 2017 29(10):1737-1741.

6. Seino S, Shinkai S, Fujiwara Y, Obuchi S, Yoshida H, Hirano H, et al. TMIG-LISA Research Group. Reference values and age and sex differences in physical performance measures for communitydwelling older Japanese: a pooled analysis of six cohort studies. PLoS One. 2014;9(6):e99487.

7. Chen WL, Peng TC, Sun YS, Yang HF, Liaw FY, Wu LW, et al. Examining the Association Between Quadriceps Strength and Cognitive Performance in the Elderly. Medicine (Baltimore). 2015;94(32):e1335.

8. Ibrahim A, Singh DKA, Shahar S. 'Timed Up and Go' test: Age, gender and cognitive impairment stratified normative values of older adults. PLoS One. 2017;12(10):e0185641.

9. Makizako H, Shimada H, Doi T, Tsutsumimoto K, Lee S, Lee SC, et al. Age-dependent changes in physical performance and body composition in community-dwelling Japanese older J Cachexia Sarcopenia Muscle. 2017;8(4):607-14.

10. Omori G, Koga Y, Tanaka M, Nawata A, Watanabe H, Narumi K, et al. Quadriceps muscle strength and its relationship to radiographic knee osteoarthritis in Japanese elderly. J Orthop Sci. 2013;18(4):53642.

11. Sternäng O, Reynolds CA, Finkel D, Ernsth-Bravell M, Pedersen NL, Dahl Aslan AK. Factors associated with grip strength decline in older adults. Age Ageing. 2015;44(2):269-74.

12. Sternäng O, Reynolds CA, Finkel D, Ernsth-Bravell M, Pedersen NL, Dahl Aslan AK. Grip Strength and Cognitive Abilities: Associations in Old Age. J Gerontol B Psychol Sci Soc Sci. 2016;71(5):841-8.

13. Taekema DG, Gussekloo J, Maier AB, Westendorp RG, de Craen AJ. Handgrip strength as a predictor of functional, psychological and social health. A prospective population-based study among the oldest old. Age Ageing. 2010;39(3):331-7.

14. Taniguchi N, Matsuda S, Kawaguchi T, Tabara Y, Ikezoe T, Tsuboyama T, et al. The KSS 2011 reflects symptoms, physical activities, and radiographic grades in a Japanese Clin Orthop Relat Res. 2015;473(1):70-5.

15. Vellas BJ, Wayne SJ, Romero L, Baumgartner RN, Rubenstein LZ, Garry PJ. One-leg balance is an important predictor of injurious falls in older J Am Geriatr Soc. 1997;45(6):735-8.

16. Chan OY, van Houwelingen AH, Gussekloo J, Blom JW, den Elzen WP. Comparison of quadriceps strength and handgrip strength in their association with health outcomes in older adults in primary care. Age (Dordr). 2014;36(5):9714. 
17. Ishii Y, Noguchi H, Sato J, Ishii H, Takayama S, Toyabe SI. Life expectancy of osteoarthritic patients after primary total knee arthroplasty. J Clin Orthop Trauma. 2017 Nov;8(Suppl 2):S57-S61

18. Sullivan KJ, Husak LE, Altebarmakian M, Brox WT. Demographic factors in hip fracture incidence and mortality rates in California, 2000-2011. J Orthop Surg Res. 2016 Jan 8;11:4.

19. Arinzon Z, Shabat S, Peisakh A, Gepstein R, Berner YN. Gender differences influence the outcome of geriatric rehabilitation following hip fracture. Arch Gerontol Geriatr. 2010 Jan-Feb;50(1):86-91.

20. Endo Y, Aharonoff GB, Zuckerman JD, Egol KA, Koval KJ. Gender differences in patients with hip fracture: a greater risk of morbidity and mortality in men. J Orthop Trauma. 2005 Jan;19(1):29-35.

21. Folstein MF, Folstein SE, McHugh PR. "Mini-mental state". A practical method for grading the cognitive state of patients for the clinician. J Psychiatr Res. 1975;12(3):189-98.

22. Nara M, Sugie M, Takahashi T, Koyama T, Sengoku R, Fujiwara $Y$, et al. Japanese version of the Montreal Cognitive Assessment cut-off score to clarify improvement of mild cognitive impairment after exercise training in community-dwelling older adults. Geriatr Gerontol Int. 2018;18(6):833-8.

23. Brown W, Birnstihl EA, Wheeler DW. 1996. Leading without Authority: An Examination of the Impact of Transformational Leadership Cooperative Extension Work Groups and Teams. Journal of Extension, 34:5FEA3.

24. Hartman YAW, Karssemeijer EGA, van Diepen LAM, Olde Rikkert MGM, Thijssen DHJ. Dementia Patients Are More Sedentary and Less Physically Active than Age- and Sex-Matched Cognitively Healthy Older Adults. Dement Geriatr Cogn Disord. 2018;46(1-2):81-9.

25. Matsueda $M$, Ishii $Y$. The relationship between dementia score and ambulatory level after hip fracture in the elderly. Am J Orthop (Belle Mead NJ). 2000;29(9):691-3.

26. Vogt L, Wieland K, Bach M, Himmelreich H, Banzer W. Cognitive status and ambulatory rehabilitation outcome in geriatric patients. J Rehabil Med. 2008;40(10):876-8.

27. Umegaki H, Makino T, Yanagawa M, Nakashima H, Kuzuya M, Sakurai T, et al. Maximum gait speed is associated with a wide range of cognitive functions in Japanese older adults with a Clinical Dementia Rating of 0.5. Geriatr Gerontol Int. 2018;18(9):1323-9.

28. Narumi K, Funaki Y, Yoshimura N, Muraki S, Omori G, Nawata A, et al. Quadriceps muscle strength reference value as index for functional deterioration of locomotive organs: Data from 3617 men and women in Japan. J Orthop Sci. 2017;22(4):765-70.

29. Ishii Y, Noguchi H, Sato J, Sakurai T, Toyabe SI Quadriceps strength impairment in the mid- to longterm follow-up period after TKA. Knee Surg Sports Traumatol Arthrosc. 2017;25(11):3372-7.

30. Michikawa T, Nishiwaki Y, Takebayashi T, Toyama Y. One-leg standing test for elderly populations. J Orthop Sci. 2009;14(5):675-85.

31. Seichi A, Hoshino Y, Doi T, Akai M, Tobimatsu Y, Kita K, et al. Determination of the optimal cutoff time to use when screening elderly people for locomotive syndrome using the one-leg standing test (with eyes open). J Orthop Sci. 2014;19(4):620-6. 
32. Harato K, Kobayashi S, Kojima I, Sakurai A, Tanikawa H, Niki Y. Factors affecting one-leg standing time in patients with end-stage knee osteoarthritis and the age-related recovery process following total knee arthroplasty. J Orthop Surg Res. 2017;12(1):21.

33. Kellgren JH, Lawrence JS. Radiographical assessment of osteoarthritis. Ann Rheum Dis. 1957;16(4):494-502.

34. Kamide N, Takahashi-Narita K, Kawamura A, Mizuno K. Determination of the reference value and systematic bias of functional reach test in Japanese elderly people by meta-analysis. J Clin Gerontol Geriatr. 2012;3:122-6.

35. Bohannon RW, Wolfson LI, White WB. Functional reach of older adults: normative reference values based on new and published data. Physiotherapy. 2017;103(4):387-91.

36. Ando M, Kamide N. Japanese elderly persons walk faster than non-Asian elderly persons: a metaregression analysis. J Phys Ther Sci. 2015;27(11):3481-5.

37. Grande G, Triolo F, Nuara A, Welmer AK, Fratiglioni L, Vetrano DL. Measuring gait speed to better identify prodromal dementia. Exp Gerontol. 2019;124:110625.

38. Amano T, Tanaka S, Ito H, Morikawa S, Uchida S. Quantifying walking ability in Japanese patients with knee osteoarthritis: Standard values derived from a multicenter study. J Orthop Sci. 2018;23(6):1027-31.

39. Panel on Prevention of Falls in Older Persons. American Geriatrics Society and British Geriatrics Society: Summary of the Updated American Geriatrics Society/British Geriatrics Society clinical practice guideline for prevention of falls in older persons. J Am Geriatr Soc. 2011;59(1):148-57.

40. Barry E, Galvin R, Keogh C, Horgan F, Fahey T. Is the Timed Up and Go test a useful predictor of risk of falls in community dwelling older adults: a systematic review and meta-analysis. BMC Geriatr. 2014;14:14.

41. Hans D, Dargent-Molina P, Schott AM, Sebert JL, Cormier C, Kotzki PO, et al. Ultrasonographic heel measurements to predict hip fracture in elderly women: the EPIDOS prospective study. Lancet 1996;348(9026):511-4.

42. Ishii $Y$, Noguchi H, Sato J, Yoshioka K, Toyabe S. Bone quality of the calcaneus 5years after total knee arthroplasty. J Arthroplasty. 2015;30(2):196-8.

43. Yanagimoto Y, Oshida Y, Sato Y. Effects of walking on bone quality as determined by ultrasound in the elderly. Scand J Med Sci Sports 2000;10(2):103-8.

44. Yung PS, Lai YM, Tung PY, Tsui HT, Wong CK, Hung VW, et al. Effects of weight bearing and nonweight bearing exercises on bone properties using calcaneal quantitative ultrasound. $\mathrm{Br} \mathrm{J}$ Sports Med 2005;39(8):547-51.

\section{Tables}

Table 1. Patients' characteristics and physical performance measurements among Japanese men aged 80 years or older

Page $16 / 18$ 


\begin{tabular}{lc}
\hline $\mathrm{N}=120$ & Median [percentile] \\
\hline Age (years) & $84[81,86](80-94)$ \\
Body weight $(\mathrm{Kg})$ & $60[56,66](43-84)$ \\
\hline Body length $(\mathrm{cm})$ & $159[156,164](144-177)$ \\
\hline Body mass index $\left(\mathrm{Kg} / \mathrm{m}^{2}\right)$ & $24[22,25](17-33)$ \\
\hline Mini-mental state test & $26[25,29](16-30)$ \\
\hline Grip strength (kgf) & $29[25,33](12-45)$ \\
\hline weight-adjusted (kgf/kg) & $0.49[0.40,0.55](0.24-0.71)$ \\
\hline Quadriceps strength & \\
\hline Right (N) & $302[229,359](82-679)$ \\
\hline weight-adjusted (N/kg) & $4.9[3.8,6.1](1.4-10.9)$ \\
\hline Left (N) & $312[241,382](91-669)$ \\
\hline weight-adjusted (N/kg) & $5.3[3.9,6.4](1.5-10.1)$ \\
\hline One-leg standing (min) & $14[5,39](1-60)$ \\
\hline Functional reach test (cm) N=119* & $27[21,31](5-48)$ \\
\hline $5 m$ Gait speed (m/sec) & $1.2[0.9,1.3](0.3-1.9)$ \\
\hline \multicolumn{1}{c}{ Time (sec) } & $4[4,6](3-19)$ \\
\hline Maximum gait speed (m/sec) & $1.5[1.2,1.8](0.3-2.8)$ \\
\hline \multicolumn{1}{c}{ Time (sec) } & $3[3,4](2-16)$ \\
\hline Timed Up \& Go (sec) & $8[7,10](4-23)$ \\
\hline Bone Quality (Broadband Ultrasound Analysis; dB/MHz) \\
\hline Right & $57[47,64](23-109)$ \\
\hline Left & $56[44,65](16-94)$ \\
\hline
\end{tabular}

*One patient did not complete the Functional Reach Test.

Values presented as medians [interquartile range] (range).

Table 2. Spearman's rank correlation coefficients among all physical performance measurements, age, and Mini-Mental State Examination (MMSE) 


\begin{tabular}{|c|c|c|c|c|c|c|c|c|c|c|}
\hline & Age & MMSE & GSA & QSA & OLS & FRT & $\begin{array}{r}5 \mathrm{~m}- \\
\mathrm{NWS}\end{array}$ & $\begin{array}{r}5 \mathrm{~m}- \\
\mathrm{MWS} \\
\end{array}$ & TUG & BUA \\
\hline Age & 1.000 & & & & & & & & & \\
\hline \multirow[t]{2}{*}{ MMSE } & -0.081 & 1.000 & & & & & & & & \\
\hline & 0.381 & & & & & & & & & \\
\hline Grip strength adj (kgf/kg) & -0.283 & 0.165 & 1.000 & & & & & & & \\
\hline (GSA) & 0.002 & 0.072 & & & & & & & & \\
\hline Quadriceps strength adj $(\mathrm{N} / \mathrm{kg})$ & -0.220 & 0.164 & 0.457 & 1.000 & & & & & & \\
\hline$(\mathrm{QSA})$ & 0.016 & 0.076 & 0.000 & & & & & & & \\
\hline One-leg standing (sec) & -0.391 & 0.134 & 0.380 & 0.229 & 1.000 & & & & & \\
\hline (OLS) & 0.000 & 0.147 & 0.000 & 0.012 & & & & & & \\
\hline Functional Reach Test $(\mathrm{cm})$ & -0.162 & 0.205 & 0.339 & 0.329 & 0.407 & 1.000 & & & & \\
\hline$($ FRT $)$ & 0.078 & 0.025 & 0.000 & 0.000 & 0.000 & & & & & \\
\hline $5 \mathrm{~m}$ normal walking speed $(\mathrm{m} / \mathrm{sec})$ & -0.382 & 0.043 & 0.495 & 0.417 & 0.470 & 0.393 & 1.000 & & & \\
\hline$(5 \mathrm{~m}-\mathrm{NWS})$ & 0.000 & 0.644 & 0.000 & 0.000 & 0.000 & 0.000 & & & & \\
\hline $\begin{array}{c}5 \mathrm{~m} \text { Maximum walking speed } \\
(\mathrm{m} / \mathrm{sec})\end{array}$ & -0.334 & 0.063 & 0.426 & 0.479 & 0.495 & 0.507 & 0.847 & 1.000 & & \\
\hline$(5 \mathrm{~m}-\mathrm{MWS})$ & 0.000 & 0.494 & 0.000 & 0.000 & 0.000 & 0.000 & 0.000 & & & \\
\hline Timed Up \& Go (sec) & 0.366 & -0.198 & -0.414 & -0.407 & -0.518 & -0.487 & -0.685 & -0.776 & 1.000 & \\
\hline (TUG) & 0.000 & 0.031 & 0.000 & 0.000 & 0.000 & 0.000 & 0.000 & 0.000 & & \\
\hline \multirow[t]{2}{*}{$\mathrm{BUA}(\mathrm{dB} / \mathrm{MHz})$} & -0.220 & 0.055 & 0.256 & 0.206 & 0.262 & 0.220 & 0.246 & 0.300 & -0.305 & 1.000 \\
\hline & 0.016 & 0.550 & 0.005 & 0.024 & 0.004 & 0.016 & 0.007 & 0.001 & 0.001 & \\
\hline
\end{tabular}

Adj: adjusted for weight, BUA: broadband ultrasound attenuation

The upper row shows the correlation coefficient, and the bottom shows the $p$-value.

Bold numbers indicate a statistically significant correlation $(p<0.05, r \geq 0.20)$.

\section{Supplementary Files}

This is a list of supplementary files associated with this preprint. Click to download.

- STROBEchecklistv4combinedPlosMedicine1.doc 